Research Article

\title{
Synchronization of Complex Dynamical Networks on Time Scales via Pinning Control
}

\author{
Fang-Di Kong $\mathbb{D}^{1,2}$ and Jian-Ping Sun $\mathbb{D}^{1,2}$ \\ ${ }^{1}$ College of Electrical and Information Engineering, Lanzhou University of Technology, Lanzhou 730050, China \\ ${ }^{2}$ Department of Applied Mathematics, Lanzhou University of Technology, Lanzhou 730050, China \\ Correspondence should be addressed to Jian-Ping Sun; jpsun@lut.edu.cn
}

Received 10 February 2021; Accepted 26 February 2021; Published 20 March 2021

Academic Editor: Yi Qi

Copyright (C) 2021 Fang-Di Kong and Jian-Ping Sun. This is an open access article distributed under the Creative Commons Attribution License, which permits unrestricted use, distribution, and reproduction in any medium, provided the original work is properly cited.

In this paper, we are concerned with the synchronization problem of complex dynamical networks on time scales. Some pinning synchronization criteria, which combine main characteristics of time scales with main parameters of the pinning controlled network, are established. A numerical example is also included to verify the effectiveness of the results obtained.

\section{Introduction}

Many complex systems in nature and human societies can be modeled by complex dynamical networks with the nodes representing individuals in the system and the edges representing the interactions among them, such as social networks, food webs, the Internet, the World Wide Web, and neural networks (see [1] and the references therein). One of the most ubiquitous and significant phenomena in complex dynamical networks is the synchronization of all dynamical nodes in a network. Over the past decades, the synchronization has attracted considerable attention [2-9]. Control would be a necessary means to guide or force the complex dynamical network to realize synchronization if a given network is not self-synchronized or the synchronized state is not the desired one. At present, the control methods which are often used include adaptive control $[10,11]$, impulsive control [12], pinning adaptive control [13], pinning impulsive control [14], pinning feedback control [15-25], and so on [26-30]. Since pinning control only needs a small fraction of nodes to be dealt with, the synchronization of complex dynamical networks via pinning control has become a rather significant and interesting topic; see [13-27]. It is necessary to point out that most of the aforementioned discussions were aimed at the synchronization problem of continuous-time and discrete-time complex dynamical networks, respectively.

On the one hand, in some real-world systems, the interactions among individuals can take place at any time, maybe some continuous time intervals accompanying some discrete moments. On the other hand, the theory of time scales, a useful tool to deal with continuous and discrete analysis under a unified framework, was introduced by Hilger in his Ph.D. thesis [31]. With the development of the theory of time scales, the synchronization of complex dynamical networks on time scales has received increasing attention [32-40]. For example, in 2016, Liu and Zhang [35] studied the synchronization of linear complex dynamical networks on time scales via pinning impulsive control. In 2018, Lu et al. [39] considered finite-time synchronization of nonlinear complex dynamical networks on time scales via pinning impulsive control. In [40], Xiao, Lewis, and Zeng investigated event-based time-interval pinning control for complex networks on time scales.

Since time scale is an arbitrary nonempty closed subset of the real numbers, it has various forms such as the real numbers, the integers, the union of some closed intervals, and the union of some closed intervals and some discrete points. Therefore, complex dynamical networks on time scales have great complexity. Moreover, many existing 
results for continuous-time or discrete-time complex dynamical networks cannot be simply generalized to complex dynamical networks on time scales [35, 40].

Motivated greatly by the abovementioned works, in this paper, we will study a complex dynamical network on time scales by applying pinning feedback control. Some sufficient conditions are derived to guarantee the complex dynamical network to realize synchronization when it is not selfsynchronized or the synchronized state is not the desired one. The main contributions of this paper are listed as follows:

(i) In order to overcome the difficulties caused by nonlinear function $f(\cdot)$ in the complex dynamical network, we design some appropriate conditions

(ii) The pinning synchronization criteria established in our paper combine main characteristics of time scales with main parameters of the pinning controlled network (such as coupling strengths, coupling configuration matrix, and pinning feedback gain matrix)

(iii) Our results have revealed the discrepancies of the pinning synchronization between continuous-time and discrete-time complex dynamical networks

The rest of this paper is organized as follows. Some notations and supporting lemmas, and some foundational knowledge about time scales are simply enumerated in Section 2. In Section 3, the synchronization problem of a complex dynamical network on time scales is formulated. In Section 4, our main results are established. In Section 5, a numerical example is given to verify the effectiveness of the results obtained. Finally, conclusions are provided in Section 6.

\section{Preliminaries}

In this section, we will present some notations and lemmas, and some foundational knowledge on time scales which are needed later.

2.1. Notations and Supporting Lemmas. First, we define some notations as follows:

$\mathbb{N}_{0}$ is the set of all nonnegative integers

$\mathbb{Z}$ is the set of all integers

$\mathbb{R}$ is the set of all real numbers

$\mathbb{R}^{n}$ is the $n$-dimensional Euclidean space with the Euclidean norm $\|\cdot\|$

$\mathbb{R}^{m \times n}$ is the set of all $m \times n$ real matrices

$I_{n} \in \mathbb{R}^{n \times n}$ is the $n$-dimensional identity matrix

$\operatorname{diag}\left(d_{1}, d_{2}, \ldots, d_{n}\right)$ is the diagonal matrix with diagonal entries $d_{1}$ to $d_{n}$

The superscript " $T$ " stands for the transpose of a matrix

For symmetric matrix $P \in \mathbb{R}^{n \times n}, \lambda_{\text {min }}(P)$ and $\lambda_{\max }(P)$ denote the minimum and maximum eigenvalues of $P$, respectively
For symmetric matrices $P, Q \in \mathbb{R}^{n \times n}, P \geq Q(P \leq Q)$ means that $P-Q$ is positive semidefinite (negative semidefinite)

$\otimes$ denotes the Kronecker product

Lemma 1 (see [23]). Suppose $A=\left(a_{i j}\right)_{n \times n}$ is a real symmetric and irreducible matrix, in which $a_{i j} \geq 0(j \neq i)$ and $a_{i i}=-\sum_{j=1, j \neq i}^{n} a_{i j}, \quad$ and nonzero matrix $D=\operatorname{diag}\left(d_{1}, d_{2}, \ldots, d_{n}\right) \quad$ satisfies $\quad d_{i} \geq 0(1 \leq i \leq n)$ Let $B=A-D$. Then, all the eigenvalues of $B$ are less than 0 .

Lemma 2 (see [41]). If $P, Q \in \mathbb{R}^{n \times n}$ are symmetric, $x \in \mathbb{R}^{n}$ is a nonzero vector, and $0<a, b \in \mathbb{R}$, then

(1) $P+Q$ is symmetric

(2) $P^{k}$ is symmetric for $k=1,2, \ldots$,

(3) $P$ is positive definite $\Leftrightarrow-P$ is negative definite

(4) $P$ is positive definite $\Leftrightarrow$ there exists a positive definite matrix $S \in \mathbb{R}^{n \times n}$ such that $P=S^{2}$

(5) $\lambda_{\min }(P) \leq\left(x^{T} P x / x^{T} x\right) \leq \lambda_{\max }(P)$

(6) $\lambda_{\max }(a P+b Q) \leq a \lambda_{\max }(P)+b \lambda_{\max }(Q)$

Lemma 3 (see [41, 42]). For matrices $P, Q, R$, and $S$ with appropriate dimensions, we have the following properties:

(1)

$(\alpha P) \otimes Q=P \otimes(\alpha Q)=\alpha(P \otimes Q)$, where $\alpha$ is a constant

(2) $(P+Q) \otimes R=P \otimes R+Q \otimes R$

(3) $(P \otimes Q)(R \otimes S)=(P R) \otimes(Q S)$

(4) $(P \otimes Q)^{T}=P^{T} \otimes Q^{T}$

(5) If $P$ and $Q$ are symmetric, then $P \otimes Q$ is symmetric

(6) For square matrices $P$ and $Q$, every eigenvalue of $P \otimes Q$ arises as a product of eigenvalues of $P$ and $Q$

Lemma 4 (see [43]). Let $U=\left(\alpha_{i j}\right)_{N \times N}, M \in \mathbb{R}^{n \times n}$, and $x=\left(x_{1}^{T}, x_{2}^{T}, \ldots, x_{N}^{T}\right)^{T}$, where $x_{i}=\left(x_{i 1}, x_{i 2}, \ldots, x_{i n}\right)^{T} \in \mathbb{R}^{n}$ and $y=\left(y_{1}^{T}, y_{2}^{T}, \ldots, y_{N}^{T}\right)^{T}$, where $y_{i}=\left(y_{i 1}, y_{i 2}\right.$, $\left.\ldots, y_{\text {in }}\right)^{T} \in \mathbb{R}^{n}(i=1,2, \ldots, N)$. If $U=U^{T}$ and each row sum of $U$ is zero, then

$$
x^{T}(U \otimes M) y=-\sum_{1 \leq i<j \leq N} \alpha_{i j}\left(x_{i}-x_{j}\right)^{T} M\left(y_{i}-y_{j}\right) .
$$

2.2. Foundational Knowledge on Time Scales. In this section, some foundational definitions and lemmas on time scales are provided. For more details, one can refer to [44, 45].

Let $\mathbb{T}$ be a time scale; that is, $\mathbb{T}$ is a nonempty closed

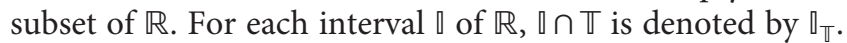
The properties of $\mathbb{T}$ are determined by the following three functions:

(1) The forward jump operator $\sigma(t)=\inf \{s \in \mathbb{T}: s>t\}, t \in \mathbb{T}$ (in this case, we put $\inf \varnothing=\sup \mathbb{T}$, where $\varnothing$ denotes the empty set) 
(2) The backward jump operator $\rho(t)=\sup \{s \in \mathbb{T}: s<t\}, t \in \mathbb{T}$ (in this case, we put $\sup \varnothing=\inf \mathbb{T}$, where $\varnothing$ denotes the empty set)

(3) The graininess function $\mu(t)=\sigma(t)-t, t \in \mathbb{T}$

For $t \in \mathbb{T}$, if $\sigma(t)>t$, we say that $t$ is right-scattered, while if $\rho(t)<t$, we say that $t$ is left-scattered. Also, if $t<\sup \mathbb{T}$ and $\sigma(t)=t$, then $t$ is called right-dense, and if $t>\inf \mathbb{T}$ and $\rho(t)=t$, then $t$ is called left-dense. The set $\mathbb{T}^{\kappa}$ is derived from the time scale $\mathbb{T}$ as follows: if $\mathbb{T}$ has a left-scattered maximum $m$, then $\mathbb{T}^{\kappa}=\mathbb{T} \backslash\{m\}$. Otherwise, $\mathbb{T}^{\kappa}=\mathbb{T}$.

Definition 1. Let $f: \mathbb{T} \longrightarrow \mathbb{R}$. Define the function $f^{\sigma}: \mathbb{T} \longrightarrow \mathbb{R}$ by $f^{\sigma}(t)=f(\sigma(t))$ for all $t \in \mathbb{T}$, i.e., $f^{\sigma}=f \circ \sigma$.

Definition 2. Assume that $f: \mathbb{T} \longrightarrow \mathbb{R}$ is a function and let $t \in \mathbb{T}^{\kappa}$. Then, $f$ is called differentiable at the point $t$ if there exists $a \theta \in \mathbb{R}$ such that for any given $\varepsilon>0$, there is an open neighborhood $U$ of $t$ such that

$$
|[f(\sigma(t))-f(s)]-\theta[\sigma(t)-s]| \leq \varepsilon|\sigma(t)-s|, \quad s \in U .
$$

In this case, $\theta$ is called the delta derivative of $f$ at the point $t$ and we denote it by $\theta=f^{\triangle}(t)$. Moreover, we say that $f$ is delta differentiable (or in short: differentiable) on $\mathbb{T}^{\kappa}$ provided $f^{\triangle}(t)$ exists for all $t \in \mathbb{T}^{\kappa}$. The function $f^{\triangle}: \mathbb{T}^{\kappa} \longrightarrow \mathbb{R}$ is called the (delta) derivative of $f$ on $\mathbb{T}^{\kappa}$. If $F^{\triangle}(t)=f(t), t \in \mathbb{T}^{\kappa}$, then for any $a, b \in \mathbb{T}$, the integral is defined as follows:

$$
\int_{a}^{b} f(t) \triangle t=F(b)-F(a) .
$$

Remark 1. If $\mathbb{T}=\mathbb{R}$, then $f^{\sigma}(t)=f(t)$ and $f^{\triangle}(t)=f^{\prime}(t)$ is the usual derivative. If $\mathbb{T}=\mathbb{Z}$, then $f^{\sigma}(t)=f(t+1)$ is the forward shift and $f^{\triangle}(t)=\Delta f(t)=f(t+1)-f(t)$ is the usual forward difference.

Lemma 5. If $f, g: \mathbb{T} \longrightarrow \mathbb{R}$ are differentiable at $t \in \mathbb{T}^{\kappa}$, then

$$
\begin{aligned}
(f g)^{\triangle}(t)= & f^{\triangle}(t) g(t)+f^{\sigma}(t) g^{\triangle}(t)=f(t) g^{\triangle}(t) \\
& +f^{\triangle}(t) g^{\sigma}(t) .
\end{aligned}
$$

Lemma 6. If $f: \mathbb{T} \longrightarrow \mathbb{R}$ is differentiable at $t \in \mathbb{T}^{\kappa}$, then $f^{\sigma}(t)=f(t)+\mu(t) f^{\triangle}(t)$.

Definition 3. A function $f: \mathbb{T} \longrightarrow \mathbb{R}$ is called rd-continuous provided it is continuous at right-dense points in $\mathbb{T}$ and its left-sided limits exist (finite) at left-dense points in $\mathbb{T}$. The set of rd-continuous functions is denoted by $C_{r d}(\mathbb{T}, \mathbb{R})$.

Definition 4 . We say that a function $p: \mathbb{T} \longrightarrow \mathbb{R}$ is regressive (positively regressive) provided

$$
1+\mu(t) p(t) \neq 0, \quad \text { for all } t \in \mathbb{T}^{\mathcal{K}}(1+\mu(t) p(t)>0 \text { for all } t \in \mathbb{T}),
$$

holds. The set of all regressive (positively regressive) and $\mathrm{rd}$ continuous functions is denoted by $\mathscr{R}(\mathbb{T}, \mathbb{R})\left(\mathscr{R}^{+}(\mathbb{T}, \mathbb{R})\right)$.

Definition 5. If $p \in \mathscr{R}(\mathbb{T}, \mathbb{R})$, then we define the exponential function by

$$
e_{p}(t, s)=\exp \left(\int_{s}^{t} \xi_{\mu(\tau)}(p(\tau)) \Delta \tau\right), \quad \text { for } s, t \in \mathbb{T}
$$

with the cylinder transformation $\xi_{h}(z)$ defined by

$$
\xi_{h}(z)= \begin{cases}\frac{1}{h} \log (1+z h), & h>0, \\ z, & h=0,\end{cases}
$$

where Log is the principal logarithm function.

Lemma 7. Let $t_{0} \in \mathbb{T}, y, f \in C_{r d}(\mathbb{T}, \mathbb{R})$, and $p \in \mathscr{R}^{+}(\mathbb{T}, \mathbb{R})$. Then,

$$
y^{\Delta}(t) \leq p(t) y(t)+f(t), \quad \text { for all } t \in \mathbb{T},
$$

implies

$$
y(t) \leq y\left(t_{0}\right) e_{p}\left(t, t_{0}\right)+\int_{t_{0}}^{t} e_{p}(t, \sigma(\tau)) f(\tau) \Delta \tau, \quad \text { for all } t \in \mathbb{T} .
$$

Lemma 8 (see [40]). For fixed $t_{0} \in \mathbb{T}$, if $p<0$ and $p \in \mathscr{R}^{+}(\mathbb{T}, \mathbb{R})$, then $e_{p}\left(t, t_{0}\right) \longrightarrow 0$ as $t \longrightarrow \infty, t \in \mathbb{T}$.

Definition 6. Let $A$ be an $m \times n$-matrix-valued function on $\mathbb{T}$. We say that $A$ is rd-continuous on $\mathbb{T}$ if each entry of $A$ is rd-continuous on $\mathbb{T}$. We say that $A$ is differentiable on $\mathbb{T}$ provided each entry of $A$ is differentiable on $\mathbb{T}$. In this case, we put $A^{\triangle}=\left(a_{i j}^{\triangle}\right)_{1 \leq i \leq m, 1 \leq j \leq n}$, where $A=\left(a_{i j}\right)_{1 \leq i \leq m, 1 \leq j \leq n}$.

Lemma 9. If $A$ is differentiable at $t \in \mathbb{T}^{\kappa}$, then $A^{\sigma}(t)=A(t)+\mu(t) A^{\triangle}(t)$.

Lemma 10. Suppose $A$ and $B$ are differentiable $n \times n$-matrixvalued functions on $\mathbb{T}$. Then,

(1) $(A+B)^{\triangle}=A^{\triangle}+B^{\triangle}$

(2) $(\alpha A)^{\triangle}=\alpha A^{\triangle}$ if $\alpha$ is constant

(3) $(A B)^{\triangle}=A^{\triangle} B+A^{\sigma} B^{\triangle}=A B^{\triangle}+A^{\triangle} B^{\sigma}$

Definition 7. An $n \times n$-matrix-valued function $A$ on $\mathbb{T}$ is called regressive (with respect to $\mathbb{T}$ ) provided $I_{n}+\mu(t) A(t)$ is invertible for all $t \in \mathbb{T}^{\kappa}$, and the class of all such regressive and rd-continuous functions is denoted by $\mathscr{R}\left(\mathbb{T}, \mathbb{R}^{n \times n}\right)$.

\section{Problem Formulations}

In the remainder of this paper, we always assume that $\mathbb{T}$ is a time scale with $0 \in \mathbb{T}$ and $\sup \mathbb{T}=\infty$.

Suppose that a complex dynamical network on $\mathbb{1}$ consists of $N$ identical nodes, with each node being an 
n-dimensional dynamical system. This complex dynamical network can be described as

$$
\begin{aligned}
x_{i}^{\triangle}(t)= & f\left(x_{i}(t)\right)+\sum_{j=1, j \neq i}^{N} c_{i j} g_{i j} \\
& \Gamma\left(x_{j}(t)-x_{i}(t)\right), \quad t \in[0, \infty)_{\mathbb{T}}, i=1,2, \ldots, N,
\end{aligned}
$$

where $x_{i}(t)=\left(x_{i 1}(t), x_{i 2}(t), \ldots, x_{i n}(t)\right)^{T} \in \mathbb{R}^{n}$ is the state vector of the $i$ th node at time $t, x_{i}^{\triangle}$ is the delta derivative of $x_{i}$ on $[0, \infty)_{\mathbb{V}}, f: \mathbb{R}^{n} \longrightarrow \mathbb{R}^{n}$ is a vector function, the constant $c_{i j}>0(1 \leq i, j \leq N, i \neq j)$ represents the coupling strength between node $i$ and node $j, \Gamma \in \mathbb{R}^{n \times n}$ is the inner coupling matrix, and the coupling configuration matrix $G=\left(g_{i j}\right)_{N \times N}$ represents the topological structure of the complex dynamical network and is defined as follows: if there exists a connection between node $i$ and node $j(i \neq j)$, then $g_{i j}=g_{j i}=1$; otherwise, $g_{i j}=g_{j i}=0$; the diagonal elements of $G$ are defined as

$$
g_{i i}=-\sum_{j=1, j \neq i}^{N} g_{i j}=-\sum_{j=1, j \neq i}^{N} g_{j i}, \quad i=1,2, \ldots, N,
$$

and $c_{i i}(1 \leq i \leq N)$ satisfies

$$
c_{i i} g_{i i}+\sum_{j=1, j \neq i}^{N} c_{i j} g_{i j}=c_{i i} g_{i i}+\sum_{j=1, j \neq i}^{N} c_{j i} g_{j i}=0, \quad i=1,2, \ldots, N .
$$

Then, network (10) can be equivalently written in the following form:

$$
x_{i}^{\triangle}(t)=f\left(x_{i}(t)\right)+\sum_{j=1}^{N} c_{i j} g_{i j} \Gamma x_{j}(t), \quad t \in[0, \infty)_{\mathbb{T}}, i=1,2, \ldots, N .
$$

In what follows, we always assume that network (13) is connected in the sense of having no isolated clusters, which means that the symmetric matrix $G$ is irreducible, and the following condition is satisfied:

$$
c_{i i} g_{i i} \Gamma \in \mathscr{R}\left(\mathbb{T}, \mathbb{R}^{n \times n}\right), \quad i=1,2, \ldots, N .
$$

Now, our goal is to control network (13) onto a solution of the uncoupled system

$$
s^{\triangle}(t)=f(s(t)), \quad t \in[0, \infty)_{\mathbb{T}},
$$

that is,

$$
\lim _{t \longrightarrow \infty}\left\|x_{i}(t)-s(t)\right\|=0, \quad i=1,2, \ldots, N .
$$

Here, we assume that $f$ is continuous and of such a nature that existence and uniqueness of solutions to dynamic equation (15) subject to $s(0)=s_{0}$ as well as their dependence on initial values is guaranteed.

To achieve the goal, we apply the pinning control strategy on a fraction of the nodes in network (13). Without loss of generality, let the first $l$ nodes be selected to be pinned. The pinning controlled network can be described as follows:

$$
\begin{aligned}
& x_{i}^{\triangle}(t)=f\left(x_{i}(t)\right)+\sum_{j=1}^{N} c_{i j} g_{i j} \Gamma x_{j}(t)+u_{i}(t), \quad t \in[0, \infty)_{\mathbb{T}}, i=1,2, \ldots, l, \\
& x_{i}^{\triangle}(t)=f\left(x_{i}(t)\right)+\sum_{j=1}^{N} c_{i j} g_{i j} \Gamma x_{j}(t), \quad t \in[0, \infty)_{\mathbb{T}}, i=l+1, l+2, \ldots, N,
\end{aligned}
$$

where $\quad u_{i}(t)=-c_{i i} d_{i} \Gamma\left(x_{i}(t)-s(t)\right), \quad t \in[0, \infty)_{\mathbb{T}}, \quad d_{i}>0$, and $c_{i i}\left(g_{i i}-d_{i}\right) \Gamma \in \mathscr{R}\left(\mathbb{T} ; \mathbb{R}^{n \times n}\right), i=1,2, \ldots, l$.

$$
\dot{x}_{i}(t)=f\left(x_{i}(t)\right)+\sum_{j=1}^{N} c_{i j} g_{i j} \Gamma x_{j}(t), \quad t \in[0, \infty), i=1,2, \ldots, N,
$$

Remark 2. If $\mathbb{T}=\mathbb{R}$, then network (13) is reduced to the continuous-time network:

and pinning controlled network (17) can be described by

$$
\begin{aligned}
& \dot{x}_{i}(t)=f\left(x_{i}(t)\right)+\sum_{j=1}^{N} c_{i j} g_{i j} \Gamma x_{j}(t)+u_{i}(t), \quad t \in[0, \infty), i=1,2, \ldots, l, \\
& \dot{x}_{i}(t)=f\left(x_{i}(t)\right)+\sum_{j=1}^{N} c_{i j} g_{i j} \Gamma x_{j}(t), \quad t \in[0, \infty), i=l+1, l+2, \ldots, N,
\end{aligned}
$$

where $\quad u_{i}(t)=-c_{i i} d_{i} \Gamma\left(x_{i}(t)-s(t)\right), t \in[0, \infty)$, $d_{i}>0, i=1,2, \ldots, l$, and $s(t)$ is a solution of the system

$$
\dot{s}(t)=f(s(t)), \quad t \in[0, \infty) .
$$


Remark 3. If $\mathbb{T}=\mathbb{Z}$, then network (13) is reduced to the discrete-time network:

$$
\Delta x_{i}(t)=f\left(x_{i}(t)\right)+\sum_{j=1}^{N} c_{i j} g_{i j} \Gamma x_{j}(t), \quad t \in \mathbb{N}_{0}, i=1,2, \ldots, N,
$$

$$
\begin{aligned}
& \Delta x_{i}(t)=f\left(x_{i}(t)\right)+\sum_{j=1}^{N} c_{i j} g_{i j} \Gamma x_{j}(t)+u_{i}(t), \quad t \in \mathbb{N}_{0}, i=1,2, \ldots, l, \\
& \Delta x_{i}(t)=f\left(x_{i}(t)\right)+\sum_{j=1}^{N} c_{i j} g_{i j} \Gamma x_{j}(t), \quad t \in \mathbb{N}_{0}, i=l+1, l+2, \ldots, N,
\end{aligned}
$$

where $u_{i}(t)=-c_{i i} d_{i} \Gamma\left(x_{i}(t)-s(t)\right), t \in \mathbb{N}_{0}, \quad d_{i}>0, i=1,2$, $\ldots, l$, and $s(t)$ is a solution of the system

$$
\Delta s(t)=f(s(t)), \quad t \in \mathbb{N}_{0} .
$$

Let $\quad x(t)=\left(x_{1}^{T}(t), x_{2}^{T}(t), \ldots, x_{N}^{T}(t)\right)^{T} \in \mathbb{R}^{n N}$, $F(x(t))=\left(f^{T}\left(x_{1}(t)\right), f^{T}\left(x_{2}(t)\right), \ldots, f^{T}\left(x_{N}(t)\right)\right)^{T} \in \mathbb{R}^{n N}$, $S(t)=\underbrace{\left(s^{T}(t), s^{T}(t), \ldots, s^{T}(t)\right)}_{N} \in \mathbb{R}^{n N}, \quad A=\left(c_{i j} g_{i j}\right)$ $\in \mathbb{R}^{N \times N}$, and $\left.D=\operatorname{diag}(c_{11} d_{1}, c_{22} d_{2}, \ldots, c_{l l} d_{l}, \underbrace{0, \ldots, 0}_{N-l})\right)$. Then, we can write pinning controlled network (17) as

$$
\begin{aligned}
x^{\triangle}(t)= & F(x(t))+(A \otimes \Gamma) x(t)-(D \otimes \Gamma) x(t)+(D \otimes \Gamma) S(t) \\
= & F(x(t))+[(A-D) \otimes \Gamma] x(t) \\
& +(D \otimes \Gamma) S(t), \quad t \in[0, \infty)_{\mathbb{T}},
\end{aligned}
$$

and obtain the following error dynamical network:

$$
z^{\triangleright}(t)=F(x(t))-F(S(t))+[(A-D) \otimes \Gamma] z(t), \quad t \in[0, \infty)_{\mathbb{T}},
$$

where $\quad z(t)=\left(z_{1}^{T}(t), z_{2}^{T}(t), \ldots, z_{N}^{T}(t)\right)^{T} \in \mathbb{R}^{n N}$, $z_{i}(t)=x_{i}(t)-s(t) \in \mathbb{R}^{n}(i=1,2, \ldots, N), \quad$ and $F(S(t))=\underbrace{\left(f^{T}(s(t)), f^{T}(s(t)), \ldots, f^{T}(s(t))\right)}{ }^{T} \in \mathbb{R}^{n N}$.

By [17], we know that $\stackrel{N}{A}$ is a symmetric and irreducible matrix. So, it follows from Lemmas 1 and 2 that $\lambda_{\max }(A-$ $D)<0$ and $(A-D)^{2}$ is symmetric positive definite.

\section{Pinning Synchronization Criteria for Complex Dynamical Networks on Time Scales}

To derive the main results, first, we introduce a definition.

Definition 8 (see $[2,17,46]$ ). A function $\phi: \mathbb{R}^{n} \longrightarrow \mathbb{R}^{n}$ is said to be increasing if

$$
(x-y)^{T}(\phi(x)-\phi(y)) \geq 0, \quad \text { for all } x, y \in \mathbb{R}^{n} .
$$

Throughout this section, we always assume that the function $f: \mathbb{R}^{n} \longrightarrow \mathbb{R}^{n}$ satisfies Lipschitz condition; that is, there exists a constant $L>0$ such that $\|f(x)-f(y)\| \leq$ $L\|x-y\|$ holds for any $x, y \in \mathbb{R}^{n}$.

Theorem 1. Suppose that there exists a constant $\mu^{*} \geq 0$ such that $\mu(t) \leq \mu^{*}$ for all $t \in \mathbb{T}, \Gamma$ is symmetric and $\Gamma f(\cdot)$ is increasing. Then, the pinning controlled network (17) is synchronized, if there exists a constant function $\alpha \in \mathscr{R}^{+}(\mathbb{T},(-\infty, 0))$ such that

$$
2 L I_{n N}+2(A-D) \otimes \Gamma+\mu^{*}\left[L^{2} I_{n N}+(A-D)^{2} \otimes \Gamma^{2}\right] \leq \alpha I_{n N}
$$

holds.

Proof. Construct the Lyapunov function $V(t)=z^{T}(t) z(t), t \in[0, \infty)_{\mathbb{T}}$. In view of Lemmas $3,5,6,9$, and 10 , we can obtain the $\Delta$-derivative of $V(t)$ along the trajectory (25):

$$
\begin{aligned}
V^{\triangle}(t)= & {\left[\left(z^{T}(t)\right)^{\triangle} z(t)+\left(z^{T}\right)^{\sigma}(t) z^{\triangle}(t)\right] } \\
= & \left(z^{T}(t)\right)^{\triangle} z(t)+\left(z^{T}(t)+\mu(t)\left(z^{T}(t)\right)^{\triangle}\right) z^{\triangle}(t) \\
= & 2 z^{T}(t) z^{\triangle}(t)+\mu(t)\left(z^{\triangle}(t)\right)^{T} z^{\triangle}(t) \\
\leq & 2 z^{T}(t) z^{\triangle}(t)+\mu^{*}\left(z^{\triangle}(t)\right)^{T} z^{\triangle}(t) \\
= & 2 z^{T}(t)\{(F(x(t))-F(S(t)))+[(A-D) \otimes \Gamma] z(t)\}+\mu^{*}\left\{(F(x(t))-F(S(t)))^{T}\right. \\
& \left.+([(A-D) \otimes \Gamma] z(t))^{T}\right\}\{(F(x(t))-F(S(t)))+[(A-D) \otimes \Gamma] z(t)\} \\
= & V_{1}(t)+V_{2}(t)+\mu^{*}\left[V_{3}(t)+V_{4}(t)+V_{5}(t)\right], \quad t \in[0, \infty)_{\mathbb{T}},
\end{aligned}
$$


where

$$
\begin{aligned}
& V_{1}(t)=2 z^{T}(t)(F(x(t))-F(S(t))), \\
& V_{2}(t)=2 z^{T}(t)[(A-D) \otimes \Gamma] z(t), \\
& V_{3}(t)=(F(x(t))-F(S(t)))^{T}(F(x(t))-F(S(t))), \\
& V_{4}(t)=2 z^{T}(t)[(A-D) \otimes \Gamma](F(x(t))-F(S(t))), \\
& V_{5}(t)=z^{T}(t)\left[(A-D)^{2} \otimes \Gamma^{2}\right] z(t) .
\end{aligned}
$$

On the one hand, since $f$ satisfies Lipschitz condition, we get

$$
\begin{aligned}
V_{1}(t) & =2 z^{T}(t)(F(x(t))-F(S(t))) \\
& =2 \sum_{i=1}^{N} z_{i}^{T}(t)\left(f\left(x_{i}(t)\right)-f(s(t))\right) \\
& \leq 2 \sum_{i=1}^{N}\left\|z_{i}^{T}(t)\right\|\left\|f\left(x_{i}(t)\right)-f(s(t))\right\| \\
& =2 \sum_{i=1}^{N} \|\left(x_{i}(t)-(s(t))^{T}\|\| f\left(x_{i}(t)\right)-f(s(t)) \|\right. \\
& \leq 2 L \sum_{i=1}^{N}\left\|x_{i}(t)-s(t)\right\|^{2} \\
& =2 L \sum_{i=1}^{N}\left(x_{i}(t)-s(t)\right)^{T}\left(x_{i}(t)-s(t)\right) \\
& =2 L z^{T}(t) z(t), \quad t \in[0, \infty)_{\mathbb{T}}, \\
& \leq L^{2} \sum_{i=1}^{N}\left\|\left(x_{i}(t)\right)-(s(t))\right\|^{2} \\
& =L^{2} \sum_{i=1}^{N}\left(x_{i}(t)\right)-(s(t))^{T}\left(x_{i}(t)\right)-(s(t)) \\
& =(F(x(t))-F(S(t)))^{T}(F(x(t))-F(s(t))) \\
& =\sum_{i=1}^{N}\left(f\left(x_{i}(t)\right)-f(s(t))\right)^{T}\left(f\left(x_{i}(t)\right)-f(s(t))\right) \\
& \sum_{i=1} \|\left(f\left(x_{i}(t)\right)-f(s(t)) \|^{2}\right. \\
&
\end{aligned}
$$

On the other hand, since $\Gamma f(\cdot)$ is increasing, by Lemma 4 , we have

$$
\begin{aligned}
& V_{4}(t)=2 z^{T}(t)[(A-D) \otimes \Gamma](F(x(t))-F(S(t))) \\
& =2\left[z^{T}(t)(A \otimes \Gamma)(F(x(t))-F(S(t)))-z^{T}(t)\right. \\
& (D \otimes \Gamma)(F(x(t))-F(S(t)))] \\
& =-2\left\{\sum_{1 \leq i<j \leq N} c_{i j} g_{i j}\left(z_{i}(t)-z_{j}(t)\right)^{T}\right. \\
& \Gamma\left[\left(f\left(x_{i}(t)\right)-f(s(t))\right)-\left(f\left(x_{j}(t)\right)-f(s(t))\right)\right] \\
& \left.+\sum_{i=1}^{l} z_{i}^{T}(t) c_{i i} d_{i} \Gamma\left(f\left(x_{i}(t)\right)-f(s(t))\right)\right\} \\
& =-2\left[\sum_{1 \leq i<j \leq N} c_{i j} g_{i j}\left(x_{i}(t)-x_{j}(t)\right)^{T}\right. \\
& \Gamma\left(f\left(x_{i}(t)\right)-f\left(x_{j}(t)\right)\right) \\
& \left.+\sum_{i=1}^{l} c_{i i} d_{i}\left(x_{i}(t)-s(t)\right) \Gamma\left(f\left(x_{i}(t)\right)-f(s(t))\right)\right] \\
& =-2\left[\sum_{1 \leq i<j \leq N} c_{i j} g_{i j}\left(x_{i}(t)-x_{j}(t)\right)^{T}\right. \\
& \left(\Gamma f\left(x_{i}(t)\right)-\Gamma f\left(x_{j}(t)\right)\right) \\
& +\sum_{i=1}^{l} c_{i i} d_{i}\left(x_{i}(t)-s(t)\right)^{T} \\
& \left.\left(\Gamma f\left(x_{i}(t)\right)-\Gamma f(s(t))\right)\right], \quad \leq 0, t \in[0, \infty)_{\mathbb{T}} .
\end{aligned}
$$
that

So, it follows from (28), (30)-(32), and condition (27)

$$
\begin{aligned}
V^{\triangle}(t) & \leq 2 L z^{T}(t) z(t)+2 z^{T}(t)[(A-D) \otimes \Gamma] z(t)+\mu^{*}\left\{L^{2} z^{T}(t) z(t)+z^{T}(t)\left[(A-D)^{2} \otimes \Gamma^{2}\right] z(t)\right\} \\
& =z^{T}(t)\left\{2 L I_{n N}+2(A-D) \otimes \Gamma+\mu^{*}\left[L^{2} I_{n N}+(A-D)^{2} \otimes \Gamma^{2}\right]\right\} z(t) \\
& \leq \alpha V(t), \quad t \in[0, \infty)_{\mathbb{T}},
\end{aligned}
$$

which together with Lemma 7 implies that

$$
V(t) \leq V(0) e_{\alpha}(t, 0), \quad t \in[0, \infty)_{\mathbb{T}} .
$$

At the same time, it follows from $\alpha \in \mathscr{R}^{+}(\mathbb{T},(-\infty, 0))$ and Lemma 8 that 


$$
\lim _{t \longrightarrow \infty} e_{\alpha}(t, 0)=0 .
$$

In view of (34) and (35), we know that $V(t) \longrightarrow 0$ as $t \longrightarrow \infty$. This indicates that pinning controlled network (17) is synchronized.
Theorem 2. Suppose that there exists a constant $\mu^{*} \geq 0$ such that $\mu(t) \leq \mu^{*}$ for all $t \in \mathbb{T}, \Gamma$ is symmetric, and $\Gamma f(\cdot)$ is increasing. Then, pinning controlled network (17) is synchronized, if

$$
\beta:=\lambda_{\max }\left(2 L I_{n N}+2(A-D) \otimes \Gamma+\mu^{*}\left[L^{2} I_{n N}+(A-D)^{2} \otimes \Gamma^{2}\right]\right)<0, \quad \text { and } \beta \in \mathscr{R}^{+}(\mathbb{T}, \mathbb{R}) .
$$

Proof. Construct the Lyapunov function $V(t)=z^{T}(t) z(t), t \in[0, \infty)_{\mathbb{T}}$. Similar to the proof of Theorem 1 , we have

$$
V^{\triangle}(t) \leq z^{T}(t)\left\{2 L I_{n N}+2(A-D) \otimes \Gamma+\mu^{*}\left[L^{2} I_{n N}+(A-D)^{2} \otimes \Gamma^{2}\right]\right\} z(t), \quad t \in[0, \infty)_{\mathbb{T}} .
$$

So, by (37) and Lemma 2, we get

$$
\begin{aligned}
V^{\triangle}(t) \leq & \lambda_{\max }\left(2 L I_{n N}+2(A-D) \otimes \Gamma\right. \\
& \left.+\mu^{*}\left[L^{2} I_{n N}+(A-D)^{2} \otimes \Gamma^{2}\right]\right) z^{T}(t) z(t) \\
= & \beta z^{T}(t) z(t)=\beta V(t), \quad t \in[0, \infty)_{\mathbb{T}},
\end{aligned}
$$

which together with Lemma 7 implies that

$$
V(t) \leq V(0) e_{\beta}(t, 0), \quad t \in[0, \infty)_{\mathbb{T}} .
$$

At the same time, it follows from $\beta<0, \beta \in \mathscr{R}^{+}(\mathbb{T}, \mathbb{R})$, and Lemma 8 that

$$
\lim _{t \longrightarrow \infty} e_{\beta}(t, 0)=0 .
$$

In view of (39) and (40), we know that $V(t) \longrightarrow 0$ as $t \longrightarrow \infty$. This completes the proof.

Corollary 1. Suppose that there exists a constant $\mu^{*} \geq 0$ such that $\mu(t) \leq \mu^{*}$ for all $t \in \mathbb{T}, \Gamma$ is symmetric positive definite, and $\Gamma f(\cdot)$ is increasing. Then, pinning controlled network (17) is synchronized, if

$$
\begin{aligned}
\gamma:= & 2 L+2 \lambda_{\max }(A-D) \lambda_{\min }(\Gamma) \\
& +\mu^{*}\left[L^{2}+\lambda_{\max }\left((A-D)^{2}\right) \lambda_{\max }\left(\Gamma^{2}\right)\right] \\
& <0, \quad \text { and } \gamma \in \mathscr{R}^{+}(\mathbb{T}, \mathbb{R}) .
\end{aligned}
$$

Proof. Since $A-D$ is symmetric negative definite, $\Gamma, \Gamma^{2}$, and $(A-D)^{2}$ are symmetric positive definite, and by Lemmas 2 and 3 , we get

$$
\begin{aligned}
& \lambda_{\max }\left(2 L I_{n N}+2(A-D) \otimes \Gamma+\mu^{*}\left[L^{2} I_{n N}+(A-D)^{2} \otimes \Gamma^{2}\right]\right) \\
& \leq 2 L+2 \lambda_{\max }(A-D) \lambda_{\min }(\Gamma)+\mu^{*}\left[L^{2}+\lambda_{\max }\left((A-D)^{2}\right) \lambda_{\max }\left(\Gamma^{2}\right)\right] \\
& =\gamma .
\end{aligned}
$$

Similar to the proof of Theorem 2, we can prove that pinning controlled network (17) is synchronized.

Corollary 2. Suppose that there exists a constant $\mu^{*} \geq 0$ such that $\mu(t) \leq \mu^{*}$ for all $t \in \mathbb{T}, c_{i j}=c, \Gamma=I_{n}$, and $f(\cdot)$ is increasing. Then, pinning controlled network (17) is synchronized, if

$$
\begin{aligned}
\xi:= & 2 L+2 c \lambda_{\max }\left(G-D_{1}\right) \\
& +\mu^{*}\left[L^{2}+c^{2} \lambda_{\max }\left(\left(G-D_{1}\right)^{2}\right)\right]<0, \text { and } \xi \in \mathscr{R}^{+}(\mathbb{T}, \mathbb{R}),
\end{aligned}
$$

where $D_{1}=\operatorname{diag}(d_{1}, d_{2}, \ldots, d_{l}, \underbrace{0, \ldots, 0)}_{N-l}$.

When $\mathbb{T}=\mathbb{R}$, Theorem 2 yields the following result immediately.

Corollary 3. Let $\Gamma$ be symmetric. Then, pinning controlled network (19) is synchronized if

$$
\lambda_{\max }\left(L I_{n N}+(A-D) \otimes \Gamma\right)<0 .
$$

Corollary 4. Let $c_{i j}=c$ and $\Gamma$ be symmetric positive definite. Then, pinning controlled network (19) is synchronized if

$$
c>\frac{-L}{\lambda_{\max }\left(G-D_{1}\right) \lambda_{\min }(\Gamma)},
$$

where $D_{1}=\operatorname{diag}(d_{1}, d_{2}, \ldots, d_{l}, \underbrace{0, \ldots, 0)}_{N-l}$. 
Proof. In view of Lemma 1 , it is easy to know that $\lambda_{\max }\left(G-D_{1}\right)<0$. Now, the result follows from Lemmas 2, 3, and Corollary 3.

When $\mathbb{T}=\mathbb{Z}$, Theorem 2 yields the following result immediately.

Corollary 5. Suppose that $\Gamma$ is symmetric and $\Gamma f(\cdot)$ is increasing. Then, pinning controlled network (22) is synchronized if

$$
-1<\lambda_{\max }\left((2 L+L)^{2} I_{n N}+2(A-D) \otimes \Gamma+(A-D)^{2} \otimes \Gamma^{2}\right)<0 .
$$

Corollary 6. Suppose that $c_{i j}=c, \Gamma$ is symmetric positive definite, and $\Gamma f(\cdot)$ is increasing. Then, pinning controlled network (22) is synchronized if

$$
\begin{aligned}
- & 1<2 L+L^{2}+2 c \lambda_{\max }\left(G-D_{1}\right) \lambda_{\min }(\Gamma)+c^{2} \lambda_{\max } \\
& \left(\left(G-D_{1}\right)^{2}\right) \lambda_{\max }\left(\Gamma^{2}\right)<0,
\end{aligned}
$$

where $D_{1}=\operatorname{diag}(d_{1}, d_{2}, \ldots, d_{l}, \underbrace{0, \ldots, 0)}_{N-l}$.

Remark 4. By comparing Corollaries 4 and 6, we find that the pinning synchronization criteria for discrete-time complex dynamical networks are different from those for continuous-time complex dynamical networks. For example, to achieve pinning synchronization, in the discrete-time case, the coupling strength needs to have an upper bound, while there is no requirement of upper bound for the coupling strength in the continuous-time case.

Obviously, the research of the synchronization problem for complex dynamical networks on time scales is more general. On the one hand, it provides a unified framework for continuous-time and discrete-time complex dynamical networks. On the other hand, it can give us a better insight into the differences of the pinning synchronization between continuous-time and discrete-time complex dynamical networks.

\section{A Numerical Example}

To verify the effectiveness of the results established in Section 4 , we give a numerical example in this section.

Example 1. Consider the following complex dynamical network with ten nodes on $\mathbb{T}$ :

$$
\begin{aligned}
x_{i}^{\triangle}(t)= & \left(\begin{array}{l}
x_{i 1}^{\Delta}(t) \\
x_{i 2}^{\Delta}(t)
\end{array}\right)=\left(\begin{array}{l}
0.1 \tanh \left(x_{i 1}(t)\right) \\
0.1 \tanh \left(x_{i 2}(t)\right)
\end{array}\right) \\
& +\sum_{j=1}^{10} c_{i j} g_{i j} x_{j}(t), \quad t \in[0, \infty)_{\mathbb{T}}, i=1,2, \ldots, 10,
\end{aligned}
$$

where

$$
G=\left(\begin{array}{cccccccccc}
-4 & 1 & 1 & 0 & 0 & 1 & 0 & 1 & 0 & 0 \\
1 & -4 & 0 & 0 & 0 & 0 & 1 & 1 & 1 & 0 \\
1 & 0 & -4 & 0 & 0 & 1 & 1 & 1 & 0 & 0 \\
0 & 0 & 0 & -3 & 1 & 0 & 1 & 0 & 0 & 1 \\
0 & 0 & 0 & 1 & -3 & 0 & 0 & 1 & 0 & 1 \\
1 & 0 & 1 & 0 & 0 & -3 & 0 & 0 & 0 & 1 \\
0 & 1 & 1 & 1 & 0 & 0 & -5 & 1 & 1 & 0 \\
1 & 1 & 1 & 0 & 1 & 0 & 1 & -6 & 1 & 0 \\
0 & 1 & 0 & 0 & 0 & 0 & 1 & 1 & -3 & 0 \\
0 & 0 & 0 & 1 & 1 & 1 & 0 & 0 & 0 & -3
\end{array}\right) .
$$

Note that each isolated node of network (48) is a system described by

$$
s^{\triangle}(t)=\left(\begin{array}{c}
s_{1}^{\Delta}(t) \\
s_{2}^{\Delta}(t)
\end{array}\right)=\left(\begin{array}{l}
0.1 \tanh \left(s_{1}(t)\right) \\
0.1 \tanh \left(s_{2}(t)\right)
\end{array}\right), \quad t \in[0, \infty)_{\mathbb{T}} .
$$

Obviously, $G$ is a symmetric and irreducible matrix. Since $f\left(\begin{array}{l}u \\ v\end{array}\right)=\left(\begin{array}{c}0.1 \tanh (u) \\ 0.1 \tanh (v)\end{array}\right)$ and $\Gamma=I_{2}$, it is easy to know that $f(\cdot)$ satisfies Lipschitz condition with $L=0.1$ and $\Gamma f(\cdot)=f(\cdot)$ is increasing. Our objective is to synchronize network (48) onto the solution $s=(0,0)^{T}$ of system (50) by applying pinning control strategy. For convenience, let $c_{i j}=c=0.08$ in this example. Now, we consider the following three cases.

Case 1. Let $\mathbb{T}=\cup_{k=0}^{\infty}[k, k+0.6]$.

From Figure 1, we find that network (48) cannot synchronize onto $s=(0,0)^{T}$ without control.

In this case, since

$$
\mu(t)=\left\{\begin{array}{l}
0, \quad t \in \bigcup_{k=0}^{\infty}[k, k+0.6) \\
0.4, \quad t=k+0.6, \quad k \in \mathbb{N}_{0}
\end{array}\right.
$$

it is easy to verify that $c_{i i} g_{i i} \Gamma=0.08 g_{i i} I_{2} \in \mathscr{R}\left(\mathbb{T}, \mathbb{R}^{2 \times 2}\right)$. Now, we apply pinning control to network (48) with $l=5$ and feedback gain matrix $D=0.08 \operatorname{diag}(5,7,5,2,8,0,0,0,0,0)$. By direct calculations, we know that $c_{i i}\left(g_{i i}-d_{i}\right) \Gamma=0.08\left(g_{i i}-d_{i}\right) I_{2} \in \mathscr{R}\left(\mathbb{T}, \mathbb{R}^{2 \times 2}\right), i=1,2, \ldots, 5$,

$$
\begin{aligned}
\beta:= & \lambda_{\max }\left(2 L I_{n N}+2(A-D) \otimes \Gamma+\mu^{*}\left[L^{2} I_{n N}+(A-D)^{2} \otimes \Gamma^{2}\right]\right) \\
= & \lambda_{\max }\left(2 \times 0.1 I_{20}+2(0.08 G-D)\right. \\
& \left.\otimes I_{2}+0.4\left[0.1^{2} I_{20}+(0.08 G-D)^{2} \otimes I_{2}\right]\right) \\
= & -0.0023<0
\end{aligned}
$$

and $\beta \in \mathscr{R}^{+}(\mathbb{T}, \mathbb{R})$. So, all the conditions of Theorem 2 are fulfilled. Hence, it follows from Theorem 2 that network (48) can realize pinning synchronization. In fact, Figure 2 also shows that the pinning synchronization is achieved. 

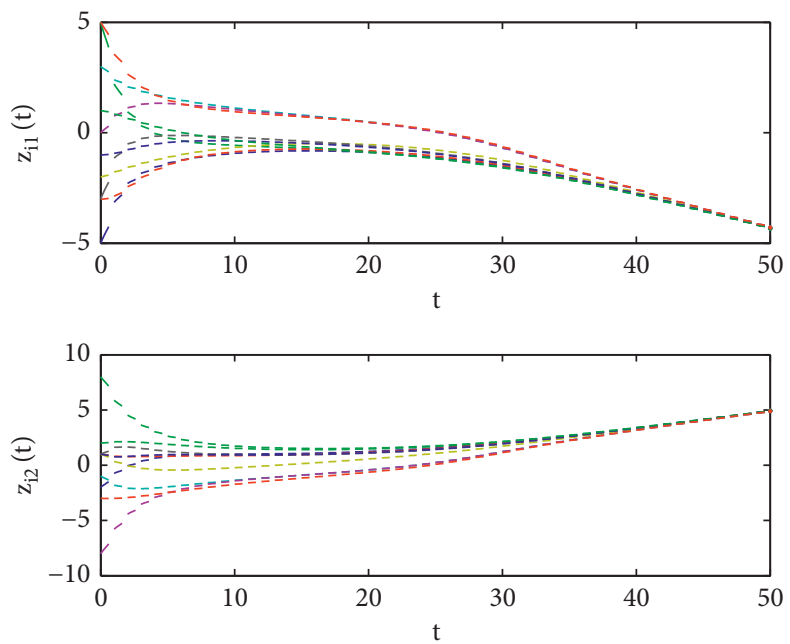

FIGURE 1: Errors $z_{i 1}$ and $z_{i 2}$ of network (48) without control, $c_{i j}=c=0.08, \mathbb{T}=\cup_{k=0}^{\infty}[k, k+0.6]$.
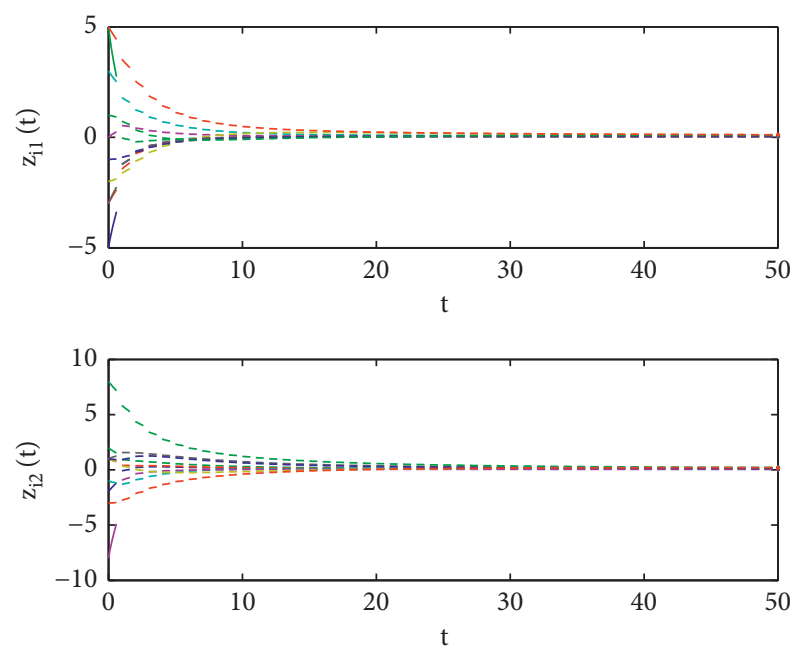

FIGURE 2: Synchronization errors $z_{i 1}$ and $z_{i 2}$ of network (48), $c_{i j}=c=0.08, \quad D=0.08 \operatorname{diag}(5,7,5,2,8,0,0,0,0,0), \quad \mathbb{T}=\cup_{k=0}^{\infty}$ $[k, k+0.6]$.

\section{Case 2. Let $\mathbb{T}=\mathbb{R}$.}

From Figure 3, we find that network (48) cannot synchronize onto $s=(0,0)^{T}$ without control.

In this case, since $\mu(t) \equiv 0$, it is easy to verify that $c_{i i} g_{i i} \Gamma=0.08 g_{i i} I_{2} \in \mathscr{R}\left(\mathbb{T}, \mathbb{R}^{2 \times 2}\right), i=1,2, \ldots, 10$. Now, we apply pinning control to network (48) with $l=6$ and feedback gain matrix $D=0.08 \operatorname{diag}(6,8,5,3,8,9,0,0,0,0)$. By direct calculations, we know that $c_{i i}\left(g_{i i}-d_{i}\right) \Gamma=0.08\left(g_{i i}-d_{i}\right) I_{2} \in \mathscr{R}\left(\mathbb{T}, \mathbb{R}^{2 \times 2}\right), i=1,2, \ldots, 6$, and $\quad \lambda_{\max }\left(L I_{n N}+(A-D) \otimes \Gamma\right)=\lambda_{\max }\left(0.1 I_{20}+\right.$ $\left.(0.08 G-D) \otimes I_{2}\right)=-0.0299<0$. So, all the conditions of Corollary 3 are fulfilled. Hence, it follows from Corollary 3 that network (48) can realize pinning synchronization. In fact, Figure 4 also shows that the pinning synchronization is achieved.

Case 3. Let $\mathbb{\square}=\mathbb{Z}$.

From Figure 5, we find that network (48) cannot synchronize onto $s=(0,0)^{T}$ without control.
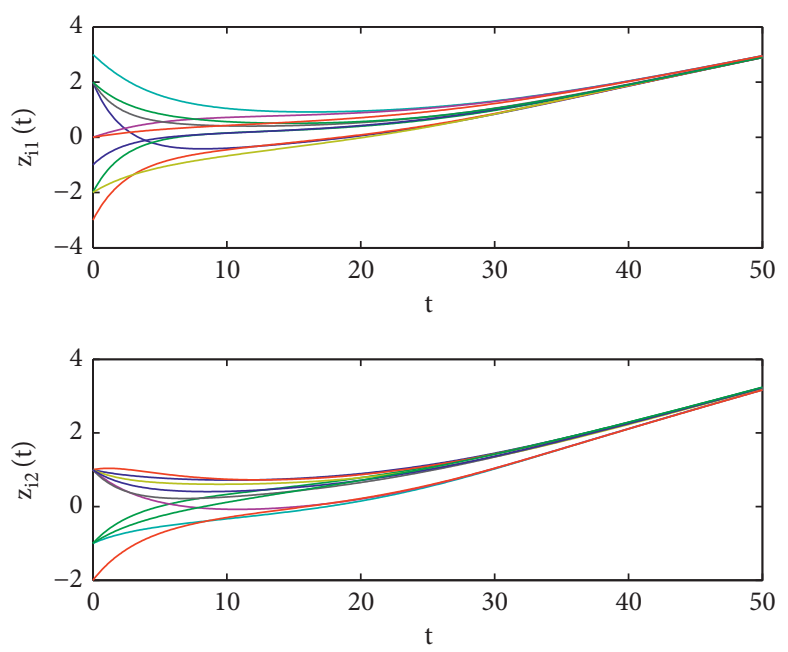

FIgURE 3: Errors $z_{i 1}$ and $z_{i 2}$ of network (48) without control, $c_{i j}=c=0.08, \mathbb{T}=\mathbb{R}$.

In this case, since $\mu(t) \equiv 0$, it is easy to verify that $c_{i i} g_{i i} \Gamma=0.08 g_{i i} I_{2} \in \mathscr{R}\left(\mathbb{T}, \mathbb{R}^{2 \times 2}\right), i=1,2, \ldots, 10$. Now, we apply pinning control to network (48) with $l=6$ and feedback gain matrix $D=0.08 \operatorname{diag}(6,8,5,3,8,9,0,0,0,0)$. By direct calculations, we know that $c_{i i}\left(g_{i i}-d_{i}\right) \Gamma=0.08\left(g_{i i}-d_{i}\right) I_{2} \in \mathscr{R}\left(\mathbb{T}, \mathbb{R}^{2 \times 2}\right), i=1,2, \ldots, 6$, and

$$
\begin{aligned}
- & 1<\lambda_{\max }\left(\left(2 L+L^{2}\right) I_{n N}+2(A-D) \otimes \Gamma+(A-D)^{2} \otimes \Gamma^{2}\right) \\
= & \lambda_{\max }\left(\left(2 \times 0.1+0.1^{2}\right) I_{20}+2(0.08 G-D)\right. \\
& \left.\otimes I_{2}+(0.08 G-D)^{2} \otimes I_{2}\right) \\
= & -0.0329<0 .
\end{aligned}
$$

So, all the conditions of Corollary 5 are fulfilled. Hence, it follows from Corollary 5 that network (48) can realize pinning synchronization. In fact, Figure 6 also shows that the pinning synchronization is achieved. 

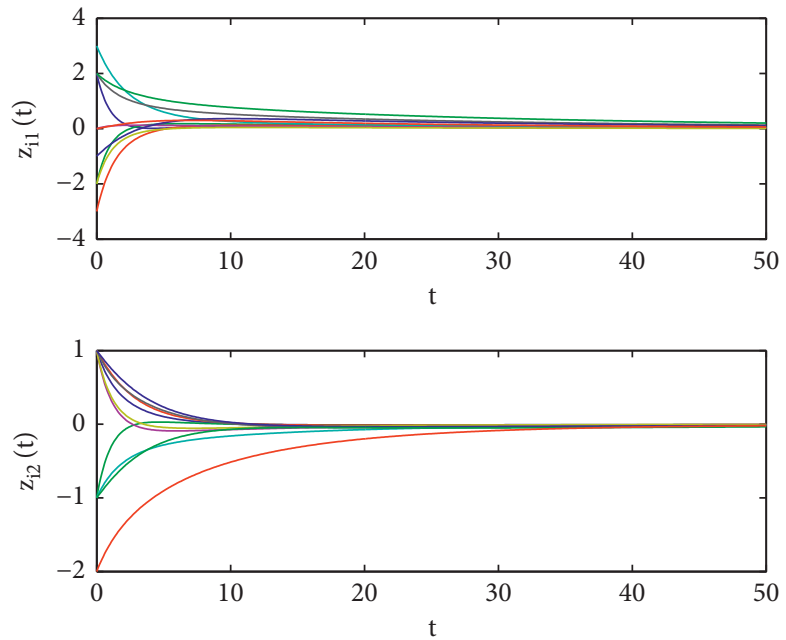

FIGURE 4: Synchronization errors $z_{i 1}$ and $z_{i 2}$ of network (48), $c_{i j}=c=0.08, D=0.08 \operatorname{diag}(6,8,5,3,8,9,0,0,0,0), \mathbb{T}=\mathbb{R}$.
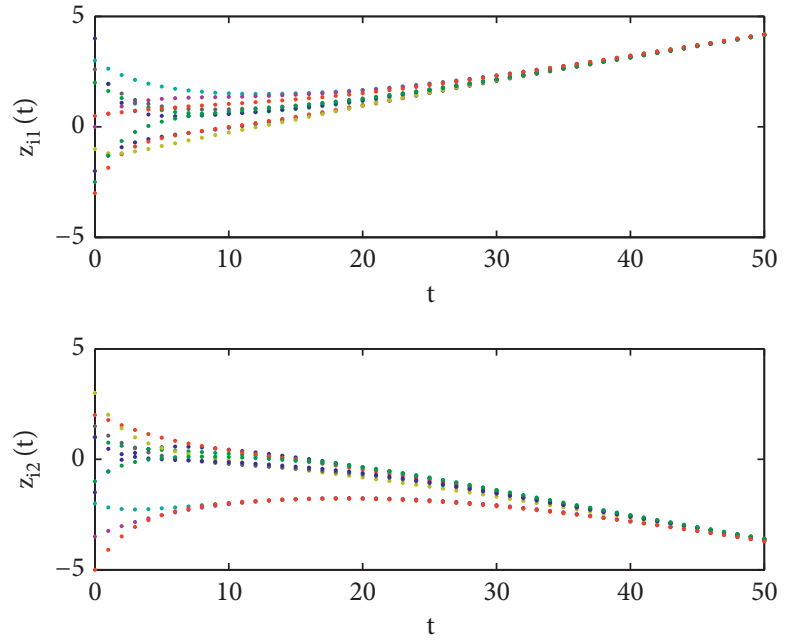

FIGURE 5: Errors $z_{i 1}$ and $z_{i 2}$ of network (48) without control, $c_{i j}=c=0.08, \mathbb{T}=\mathbb{Z}$.
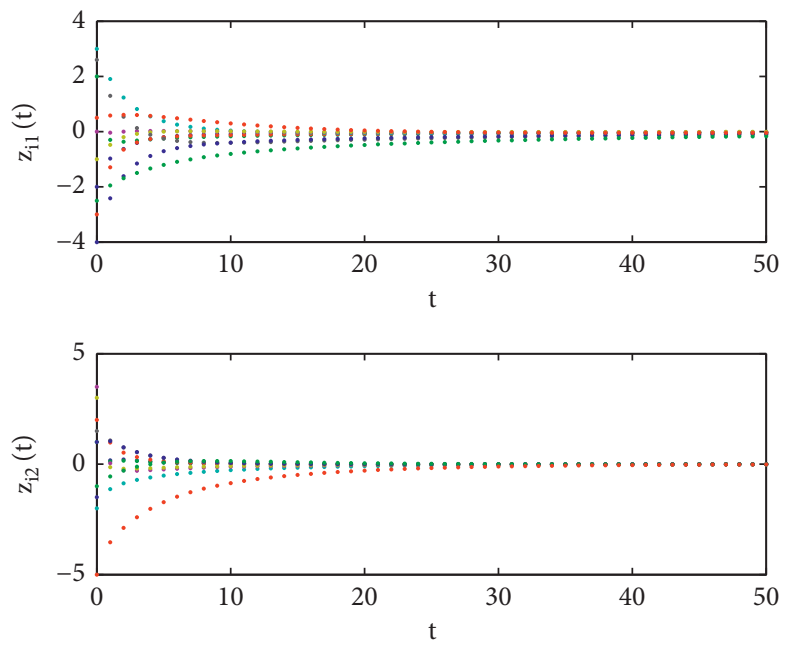

FIGURE 6: Synchronization errors $z_{i 1}$ and $z_{i 2}$ of network (48), $c_{i j}=c=0.08, D=0.08 \operatorname{diag}(6,8,5,3,8,9,0,0,0,0), \mathbb{T}=\mathbb{Z}$. 

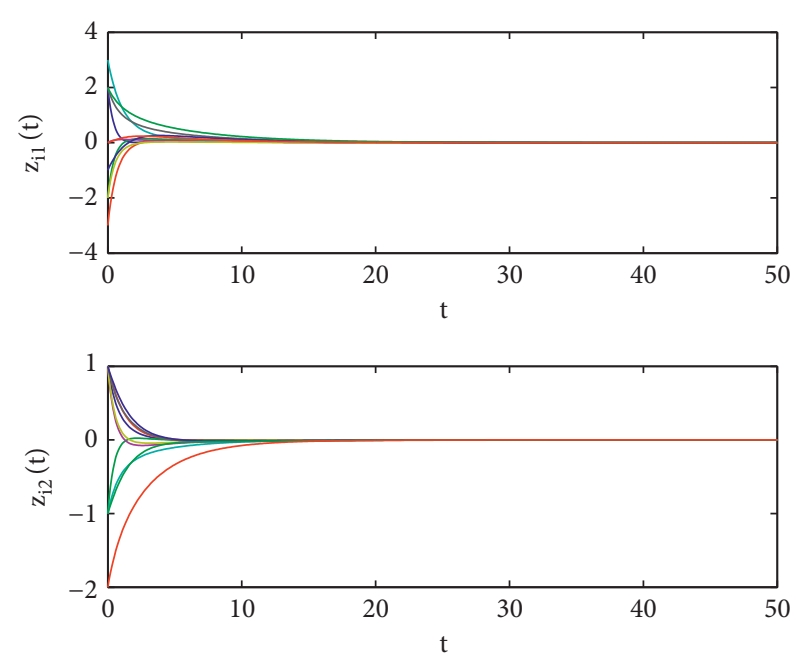

FIGURE 7: Synchronization errors $z_{i 1}$ and $z_{i 2}$ of network (48), $c_{i j}=c=0.165, D=0.165 \operatorname{diag}(6,8,5,3,8,9,0,0,0,0), \mathbb{T}=\mathbb{R}$.
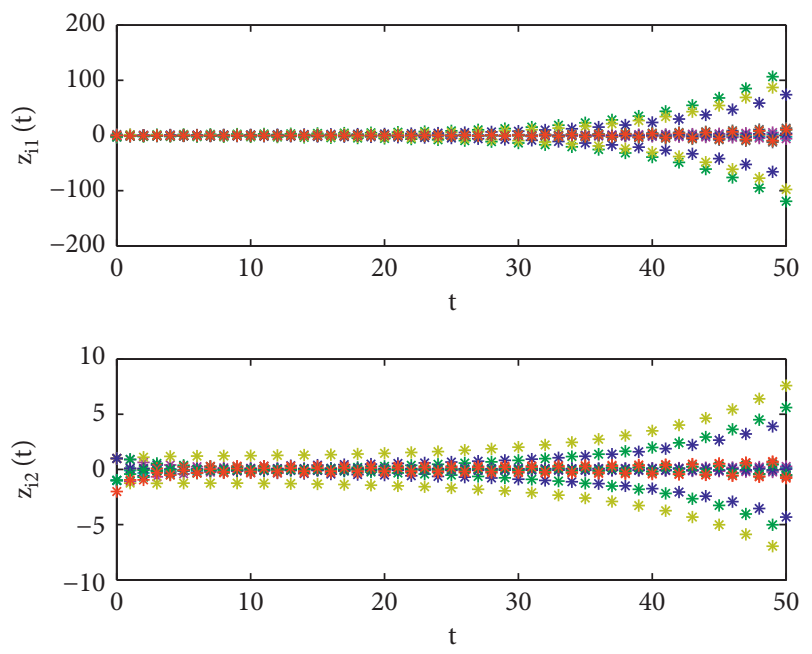

FIGURE 8: Errors $z_{i 1}$ and $z_{i 2}$ of network (48), $c_{i j}=c=0.165$, $D=0.165 \operatorname{diag}(6,8,5,3,8,9,0,0,0,0), \mathbb{T}=\mathbb{Z}$.

Remark 5. To illustrate Remark 4, we choose $c_{i j}=c=0.165$ in network (48). By numerical simulations, we find that network (48) can still realize pinning synchronization when $\mathbb{T}=\mathbb{R}$, see Figure 7 . However, Figure 8 shows that network (48) cannot realize pinning synchronization when $\mathbb{T}=\mathbb{Z}$.

\section{Conclusions}

In this paper, we have investigated the synchronization problem of a complex dynamical network on time scales by pinning control strategy. The pinning synchronization criteria established combine main characteristics of time scales with main parameters of the pinning controlled network (such as the coupling strengths, the coupling configuration matrix, and the pinning feedback gain matrix). Our results have revealed the discrepancies of the pinning synchronization between continuous-time and discrete-time complex dynamical networks. A numerical example has also been given to verify the effectiveness of the theoretical results.

\section{Data Availability}

No data were used to support this study.

\section{Conflicts of Interest}

The authors declare that there are no conflicts of interest regarding the publication of this paper.

\section{Acknowledgments}

This work was supported by the National Natural Science Foundation of China (grant no. 11661049).

\section{References}

[1] S. H. Strogatz, "Exploring complex networks," Nature, vol. 410, no. 6825, pp. 268-276, 2001.

[2] C. W. Wu, Synchronization in Coupled Chaotic Circuits and Systems, World Scientific, Singapore, 2002.

[3] C. W. Wu, Synchronization in Complex Networks of Nonlinear Dynamical Systems, World Scientific, Singapore, 2007.

[4] W. Lu and T. Chen, "Synchronization analysis of linearly coupled networks of discrete time systems," Physica D, vol. 198, no. 1-2, pp. 148-168, 2004.

[5] C. Li and G. Chen, "Synchronization in general complex dynamical networks with coupling delays," Physica A: Statistical Mechanics and its Applications, vol. 343, pp. 263-278, 2004.

[6] Z. Li and G. Chen, "Global synchronization and asymptotic stability of complex dynamical networks," IEEE Transactions on Circuits and Systems-II: Express Briefs, vol. 53, no. 1, pp. 28-33, 2006.

[7] C. P. Li, W. G. Sun, and J. Kurths, "Synchronization of complex dynamical networks with time delays," Physica A: Statistical Mechanics and its Applications, vol. 361, no. 1, pp. 24-34, 2006.

[8] X. Li and G. Chen, "Synchronization and desynchronization of complex dynamical networks: an engineering viewpoint," IEEE Transactions on Circuits and Systems-I, vol. 50, no. 11, pp. 1381-1390, 2003.

[9] Z. Wu, "Synchronization of discrete dynamical networks with non-delayed and delayed coupling," Applied Mathematics and Computation, vol. 260, pp. 57-62, 2015.

[10] J. Zhou, J. Lu, and J. Lü, "Adaptive synchronization of an uncertain complex dynamical network," IEEE Transactions on Automatic Control, vol. 51, no. 4, pp. 652-656, 2006.

[11] Q. Zhang, J. Lu, J. Lü, and K. T. Chi, "Adaptive feedback synchronization of a general complex dynamical network with delayed nodes," IEEE Transactions on Circuits and Systems-II: Express Briefs, vol. 55, no. 2, pp. 183-187, 2008.

[12] G. Zhang, Z. Liu, and Z. Ma, "Synchronization of complex dynamical networks via impulsive control, Chaos," An Interdisciplinary Journal of Nonlinear Science, vol. 17, no. 4, p. 43126, 2007.

[13] J. Zhou, J.-a. Lu, and J. Lü, "Pinning adaptive synchronization of a general complex dynamical network," Automatica, vol. 44, no. 4, pp. 996-1003, 2008.

[14] J. Lu, J. Kurths, J. Cao, N. Mahdavi, and C. Huang, "Synchronization control for nonlinear stochastic dynamical 
networks: pinning impulsive strategy," IEEE Transactions on Neural Networks and Learning Systems, vol. 23, no. 2, pp. 285-292, 2012.

[15] W. Yu, G. Chen, J. Lü, and J. Kurths, "Synchronization via pinning control on general complex networks," SIAM Journal on Control and Optimization, vol. 51, no. 2, pp. 1395-1416, 2013.

[16] X. F. Wang and G. Chen, "Pinning control of scale-free dynamical networks," Physica A, vol. 310, no. 3-4, pp. 521-531, 2002.

[17] X. Li, X. Wang, and G. Chen, "Pinning a complex dynamical network to its equilibrium," IEEE Transactions on Circuits and Systems I: Regular Papers, vol. 51, no. 10, pp. 2074-2087, 2004.

[18] T. Chen, X. Liu, and W. Lu, "Pinning complex networks by a single controller," IEEE Transactions on Circuits and Systems I: Regular Papers, vol. 54, no. 6, pp. 1317-1326, 2007.

[19] W. Yu, G. Chen, and J. Lü, "On pinning synchronization of complex dynamical networks," Automatica, vol. 45, no. 2, pp. 429-435, 2009.

[20] Q. Song and J. Cao, "On pinning synchronization of directed and undirected complex dynamical networks," IEEE Transactions on Circuits and Systems I: Regular Papers, vol. 57, no. 3, pp. 672-680, 2010.

[21] G. Chen, "Pinning control and synchronization on complex dynamical networks," International Journal of Control, Automation and Systems, vol. 12, no. 2, pp. 221-230, 2014.

[22] R. Cheng, M. Peng, and J. Zuo, "Pinning synchronization of discrete dynamical networks with delay coupling," Physica A: Statistical Mechanics and its Applications, vol. 450, pp. 444453, 2016.

[23] Z. X. Liu, Z. Q. Chen, and Z. Z. Yuan, "Pinning control of weighted general complex dynamical networks with time delay," Physica A: Statistical Mechanics and its Applications, vol. 375, no. 1, pp. 345-354, 2007.

[24] H. Su and X. Wang, Pinning Control of Complex Networked Systems: Synchronization, Consensus and Flocking of Networked Systems via Pinning, Springer, Berlin, Germany, 2013.

[25] X. Wang and H. Su, "Pinning control of complex networked systems: a decade after and beyond," Annual Reviews in Control, vol. 38, no. 1, pp. 103-111, 2014.

[26] L. Y. Xiang, Z. X. Liu, Z. Q. Chen, F. Chen, and Z. Z. Yuan, "Pinning control of complex dynamical networks with general topology," Physica A: Statistical Mechanics and its Applications, vol. 379, no. 1, pp. 298-306, 2007.

[27] H. Zhang, K. Li, and X. Fu, "On pinning control of some typical discrete-time dynamical networks," Communications in Nonlinear Science and Numerical Simulation, vol. 15, no. 2, pp. 182-188, 2010.

[28] X. Yi, R. Guo, and Y. Qi, "Stabilization of chaotic systems with both uncertainty and disturbance by the UDE-based control method," IEEE Access, vol. 8, no. 1, pp. 62471-62477, 2020.

[29] R. Xu and F. Zhang, " $\varepsilon$-nash mean-field games for general linear-quadratic systems with applications," Automatica, vol. 114, Article ID 108835, 2020.

[30] L. Liu, B. Li, and R. Guo, "Consensus control for networked manipulators with switched parameters and topologies," IEEE Access, vol. 9, pp. 9209-9217, 2021.

[31] S. Hilger, Ein Maßkettenkalkül mit anwendung auf zentrumsmannigfaltigkeiten, Ph.D. thesis, Universität Würzburg, Würzburg, Germany, 1988.

[32] Q. Cheng and J. Cao, "Synchronization of complex dynamical networks with discrete time delays on time scales," Neurocomputing, vol. 151, pp. 729-736, 2015.
[33] X. Lu, Y. Wang, and Y. Zhao, "Synchronization of complex dynamical networks on time scales via Wirtinger-based inequality," Neurocomputing, vol. 216, pp. 143-149, 2016.

[34] A. Ogulenko, "Asymptotical properties of social network dynamics on time scales," Journal of Computational and Applied Mathematics, vol. 319, pp. 413-422, 2017.

[35] X. Liu and K. Zhang, "Synchronization of linear dynamical networks on time scales: pinning control via delayed impulses," Automatica, vol. 72, pp. 147-152, 2016.

[36] M. Syed Ali and J. Yogambigai, "Synchronization of complex dynamical networks with hybrid coupling delays on time scales by handling multitude Kronecker product terms," Applied Mathematics and Computation, vol. 291, pp. 244-258, 2016.

[37] M. Syed Ali and J. Yogambigai, "Synchronization criterion of complex dynamical networks with both leakage delay and coupling delay on time scales," Neural Processing Letters, vol. 49, no. 2, pp. 453-466, 2019.

[38] Z. Huang, J. Cao, and Y. N. Raffoul, "Hilger-type impulsive differential inequality and its application to impulsive synchronization of delayed complex networks on time scales," Science China Information Sciences, vol. 61, pp. 1-3, 2018.

[39] X. Lu, X. Zhang, and Q. Liu, "Finite-time synchronization of nonlinear complex dynamical networks on time scales via pinning impulsive control," Neurocomputing, vol. 275, pp. 2104-2110, 2018.

[40] Q. Xiao, F. L. Lewis, and Z. Zeng, "Event-based time-interval pinning control for complex networks on time scales and applications," IEEE Transactions on Industrial Electronics, vol. 65, no. 11, pp. 8797-8808, 2018.

[41] H. Lütkepohl, Handbook of Matrices, Wiley, New York, NY, USA, 1996.

[42] A. N. Langville and W. J. Stewart, "The Kronecker product and stochastic automata networks," Journal of Computational and Applied Mathematics, vol. 167, no. 2, pp. 429-447, 2004.

[43] Q. Cheng and J. Cao, "Global synchronization of complex networks with discrete time delays and stochastic disturbances," Neural Computing and Applications, vol. 20, no. 8, pp. 1167-1179, 2011.

[44] M. Bohner and A. Peterson, Dynamic Equations on Time Scales: An Introduction with Applications, Birkhäuser, Boston, MA, USA, 2001.

[45] M. Bohner and A. Peterson, Advances in Dynamic Equations on Time Scales, Birkhäuser, Boston, MA, USA, 2003.

[46] L. Chua and D. Green, "A qualitative analysis of the behavior of dynamic nonlinear networks: stability of autonomous networks," IEEE Transactions on Circuits and Systems, vol. 23, no. 6, pp. 355-379, 1976. 